\title{
3 Research Square

\section{What is the normal range of obstetric shock index in the immediate postpartum period in a low resource setting?}

Johnbosco Ifunanya Nwafor ( $\sim$ nwaforjohnbosco97@gmail.com )

Alex Ekwueme Federal University Teaching Hospital https://orcid.org/0000-0001-5194-8561

Chuka Nobert Obi

Alex Ekwueme Federal University Teaching Hospital

Olisah Elvis Onuorah

Alex Ekwueme Federal University Teaching Hospital

Blessing Idzuinya Onwe

Alex Ekwueme Federal University Teaching Hospital

Chukwunenye Chukwu lbo

Alex Ekwueme Federal University Teaching Hospital

Victor Uchenna Onuchukwu

Alex Ekwueme Federal University Teaching Hospital

\section{Research Article}

Keywords: Normal range, shock index, immediate postpartum period, low resource setting.

Posted Date: April 23rd, 2020

DOI: https://doi.org/10.21203/rs.3.rs-24593/v1

License: (1) This work is licensed under a Creative Commons Attribution 4.0 International License. Read Full License

Version of Record: A version of this preprint was published at International Journal of Gynecology \& Obstetrics on July 19th, 2020. See the published version at https://doi.org/10.1002/ijgo.13297. 


\section{Abstract}

Abstract

Objective: To determine the normal range of shock index (SI), blood pressure (BP), mean arterial pressure (MAP) and heart rate (HR) among postpartum women and to compare shock index with the normal range in the current literature.

Methods: This is a prospective cohort study evaluating vital signs collected at one hour of delivery in women with normal blood loss delivered in Abakaliki, Nigeria.

Results: The median (95\% reference range) was 120 (100 - 155.8) for systolic BP, 70 (60 - 94) for diastolic BP, 90 (66.5 - 116.6) for MAP, $82(65$ - 102) for HR, and 0.69 (0.48 - 0.89) for SI. The upper limit of SI of 0.89 in this study did support the current literature suggesting a threshold of 0.9 as upper limit of normal. However, the lower limit of SI of 0.48 in this study corresponds to the lower limit of 0.5 for non-obstetric population. Over half $(56 \%)$ of the study cohorts maintained shock index values within the normal range (0.5 - 0.7) for non-obstetric population.

Conclusion: Although haemodynamic changes of pregnancy widens the range of shock index in obstetric population, $56 \%$ of the participants maintained normal shock index within the reference range for nonobstetric population. We recommend that the normal obstetric shock index range of $0.7-0.9$ in current literature be changed to $0.5-0.9$ to accommodate this lower threshold.

\section{Synopsis}

We recommend that the normal obstetric shock index range of $0.7-0.9$ in current literature be changed to 0.5 - 0.9 to accommodate the lower threshold in this study.

\section{Introduction}

About 295, 000 women died during and following pregnancy and childbirth in 2017 and $94 \%$ of these deaths occurred in low-resource settings [1]. Nigeria contributes nearly $20 \%$ of global maternal deaths [2]. Nigeria's maternal mortality ratio is over 800 maternal deaths per 100, 000 live births and most of these deaths are preventable [2]

Studies have shown that failures by healthcare providers to immediately recognise and act on signs of life-threatening conditions may have contributed to potentially avoidable direct maternal deaths [3] Therefore routine measurement of vital signs in all pregnant and postpartum women to aid early recognition of compromise is recommended [3]. Of the direct causes of maternal mortality, obstetric haemorrhage is the leading cause of maternal death with the majority occurring during labour, delivery and the immediate postpartum period [4]. All major causes of maternal death are associated with 
changes in vital signs, including blood pressure (BP) and heart rate (HR). Failure to recognise deterioration contributes to many of these avoidable deaths.

An important part of the clinical assessment of postpartum haemorrhage is an accurate estimation of blood loss. Visual estimation frequently used in clinical practice has been shown to underestimates blood loss; therefore, vital signs of systolic blood pressure (SBP) and heart rate (HR) are used to determine haemodynamic stability [5]. Thresholds of these signs are integrated into obstetric early-warning systems [6], however, physiological compensatory mechanisms of pregnancy and postpartum may mask decompensation until late in hypovolaemic shock [7]. In out-of-facility deliveries as seen in many developing countries, shock is commonly not identified until maternal loss of consciousness when it is too late for referral [8].

Shock Index (SI), the ratio of heart rate to systolic blood pressure, has been proposed as an earlier marker of compromise than conventional vital signs in non-pregnant populations [8]. It has been studied in nonspecific shock [9], trauma [10], and sepsis [11]. The normal SI range is $0.5-0.7$ for healthy adults, and an SI of $>0.9$ has been associated with increased mortality [12] In an obstetric population SI has also been proposed as a reliable marker of compromise. Despite this, the normal ranges of SI and conventional vital signs in the immediate postpartum period (i.e. within one hour of delivery) have not yet been adequately defined. To date, only few obstetric studies have defined normal SI, as 0.7-0.9 [12,13] None of these studies were done in resource-limited countries.

Therefore, the objectives of this study were to determine the normal range of obstetric SI, blood pressure (BP), mean arterial pressure (MAP) and heart rate (HR) at one hour of birth in women with normal blood loss and to compare it with the normal range in the current literature, in order to enable subsequent exploration of its use as an early warning system to identify the deteriorating postpartum woman in resource-limited settings.

\section{Materials And Methods}

Study setting: This study was conducted from April 1 to July 31, 2019 at the labour ward and the theater complex of the Alex Ekwueme Federal University Teaching Hospital (formerly known as Federal Teaching Hospital) Abakaliki is a tertiary hospital within Abakaliki, Ebonyi state, Nigeria. The hospital manages about 4500 deliveries annually and receives referral from all parts of the state and neighboring states of Benue, Enugu, Cross River and Abia as well as any part of the country. Department of Obstetrics and Gynaecology is 1 of the 10 clinical departments in the hospital, it has ten teams with each comprising consultants, senior registrars, registrars, senior house officers and house officers. The department runs gynaecological clinics, preconception, antenatal, intrapartum, and postnatal services.

Study design: This is a prospective cohort study of consenting pregnant women at term who had normal blood loss at delivery. Pregnant women attending antenatal care in the facility were informed about the study during antenatal period by the researchers. The study cohorts were women who presented in labour, being induced or scheduled for caesarean section. 
Sample size calculation: The sample size was calculated by taking variability of proportion of $20 \%$ from the average of 300 women per month who deliver in the facility. Using Open Epi software package for the determination of sample size and design effect of 1.5 at error margin of $5 \%$, the final minimum sample size was calculated as 203 at $95 \%$ confidence interval. After considering $10 \%$ drop out rate for any unpredictable events, the final required sample size was 223.3. However, 225 women were recruited for the study.

Study criteria: The study included women with term pregnancy whose estimated blood loss at delivery were within normal limit. Normal blood loss in this study was defined as estimated blood loss $<500 \mathrm{ml}$. In addition, women with medical complications such as anaemia (Haemoglobin $<10 \mathrm{~g} / \mathrm{dl}$ ) and hypertensive disorders (systolic blood pressure $\geq 140 \mathrm{mmHg}$ and/or diastolic blood pressure $\geq 90$ $\mathrm{mmHg}$ with or without proteinuria) were included in the study.

Measurement of blood loss: Blood loss at caesarean section was calculated through weighing of swabs and drapes. For participants who had vaginal delivery, once the umbilical cord was clamped and cut, a weighted impermeable leather drape measuring $60 \mathrm{~cm}$ by $45 \mathrm{~cm}$ was placed under the woman's buttocks for blood collection. Blood was collected for 1 hour. Then the drape was gently pulled out of the woman's buttocks after the client has lifted up her buttock. The drape was folded and the folded part was held in place with office pins to prevent spillage of blood. The drape with the blood was then weighed by a digital scale, with the weight recorded in grams and then converted to volume (milliliters) after the weight of the drape was subtracted assuming $1 \mathrm{~g}$ is equivalent to 1 milliliter.

Measurement of vital signs: Participants who met the inclusion criteria had their vital signs measured at 1 hour following delivery using automated device with digital display. The mean arterial pressure and shock index were calculated. This device was validated for use in pregnancy so as to minimise user error and improve accuracy.

Data collection: A specially-designed proforma was used to collect information on socio-demographic variables, obstetric characteristics, mode of delivery, estimated blood loss, blood pressure, heart rate, mean arterial pressure and shock index.

Statistical analysis: The data were analysed using SPSS Version 22.0 (IBM Corp, Armonk, New York, USA). Data were presented as mean (standard deviation) or as a percentage with range, as appropriate. Median, lower and upper quartiles and $95 \%$ reference ranges with their $90 \%$ confidence intervals were calculated for blood pressure, heart rate, mean arterial pressure and shock index. Using linear regression analysis, the association of these vital signs with predefined demographic and obstetric factors were evaluated. A $p$-value $<0.05$ was considered statistically significant.

Ethical consideration: The approval for the study was obtained from the Research and Ethics Committee of the Alex Ekwueme Federal University Teaching Hospital, Abakaliki. The study was carried out as per the guidelines given in Declaration of Helsinski 2013. 


\section{Results}

Two hundred and twenty-five women participated in this study. However, one hundred and eighty-one (181) women whose blood loss at delivery were within normal limit were included in the analysis.

The socio-demographic and obstetric characteristics of the study participants are shown in Table 1 . The age of the participants ranged from 15 to 44 years. The mean age was $30.19 \pm 5.2$. Majority $(67.9 \%)$ of the participants fall within the age range of 25 - 34 years. Mean parity was $1.79 \pm 1.9$. Five $(2.8 \%)$ participants had multiple pregnancy whereas 176 (97.2\%) of the cohorts had singleton gestation. Thirtysix $(19.9 \%)$ women had hypertensive disorders of pregnancy. Ninety-four $(51.9 \%)$ women had vaginal delivery whereas $87(48.1 \%)$ were delivered by caesarean section. Three (1.6\%) participants had anaemia (heamoglobin concentration $<10 \mathrm{~g} / \mathrm{dl}$ ) at delivery. The median (interquartile range) blood loss of the cohorts was 300 (200-400) ml.

Figures 1-5 show the histogram distribution with superimposed normal distribution curve of shock index and other vital signs in this cohort. Shock index showed normal distribution in this study unlike conventional vital signs, hence the use of median for the analysis.

The median and $95 \%$ reference ranges for the vital signs are shown in Table 2 . The median (95\% reference range) was 120 (100.0 - 155.8) for systolic BP, 70 (60.0 - 94.0) for diastolic BP, 90 (66.5 - 116.6) for MAP, $82(65$ - 102) for HR, and 0.69 (0.48 - 0.89) for SI.

Linear logistic regression analysis showed significant association between shock index and hypertensive disorders of pregnancy $(p<0.001)$ (Table 3). Shock index decreased by 0.1 with a unit increase in blood pressure, mainly due to rise in systolic BP rather than decrease in HR, equating to median SI decreasing from 0.69 to 0.59 . No other factors were significantly associated with SI in the linear regression model.

Hypertensive disorders of pregnancy $(p<0.001)$ and multiple gestation $(p=0.023)$ were significantly associated with systolic blood pressure in the regression analysis (Table 4). Systolic blood pressure increased by $19 \mathrm{mmHg}$ in the study cohorts with multiple gestation, equating to median systolic BP rising from $120 \mathrm{mmHg}$ to $139 \mathrm{mmHg}$. There were no other factors significantly associated with systolic BP in the regression analysis.

There were significant association between age $(p=0.029)$, multiple pregnancy $(p=0.005)$ and hypertensive disorders of pregnancy $(p<0.001)$ and diastolic BP (Table 5). Diastolic BP increased by 0.3 with a unit change in maternal age, equating to median diastolic BP rising from $70 \mathrm{mmHg}$ to $73 \mathrm{mmHg}$.

In this study, no other sociodemographic and clinical factors were significantly associated with heart rate except hypertensive disorders of pregnancy $(p=0.001)$ (Table 6$)$. The heart rate increased by 6 with a unit rise in blood pressure, which translates to a change in median heart rate from $82 \mathrm{bpm}$ to $88 \mathrm{bpm}$.

Like diastolic BP, MAP was significantly associated with maternal age $(p=0.027)$, multiple gestation ( $p=$ 0.003 ) and hypertensive disorders of pregnancy $(p<0.001)$ (Table 7$)$. No other factors was associated 
with MAP in these cohorts.

\section{Discussion}

This study prospectively evaluated the normal range of BP, HR, MAP and SI at the first hour of delivery. This study is unique in that it is the first study to prospectively determine the normal range of shock index and other vital signs in the immediate postpartum period. Previous studies evaluating normal ranges of shock index were either retrospective studies or secondary analysis of prospective observational studies [12-14] and some have used time points beyond the first hour despite the World Health Organization recommendation on the need for adequate monitoring of postpartum women to commence within the first hour of birth [15]. In addition, this is the first study to evaluate normal ranges of shock index in a lowresource setting as values from other studies were derived from women in high income countries.

In the maternity units worldwide, monitoring of vital signs is used routinely to evaluate the haemodynamic status of postpartum women following delivery. It is important to define normal ranges of shock index and conventional vital signs in the immediate postpartum period as pregnant and recently pregnant women may decompensate relatively late following haemorrhage and sepsis compared to other adults due to haemodynamic changes associated with pregnancy. This is particularly necessary in a resource-limited settings like Nigeria where most deliveries occur in remote areas with limited birth attendants. Therefore, defining normal range of shock index for our postpartum women population would assist in early detection of deteriorating women after delivery for urgent referral to higher healthcare facility for necessary intervention.

In this study, the lower $95 \%$ reference range for systolic BP $(100 \mathrm{mHg})$ corresponds with the lower limit of systolic BP (100 mmHg) and the upper $95 \%$ reference range for HR (102 bpm) corresponds with the upper limit of HR (100 bpm) for the recognition of shock on the currently recommended MEOWS chart. Also, the upper $95 \%$ reference ranges for systolic and diastolic BP $(155 \mathrm{mmHg}$ and $94 \mathrm{mmHg}$, respectively) correspond well with the early warning chart hypertensive triggers $(150 \mathrm{mmHg}$ and $90 \mathrm{mmHg}$ ). This finding is similar to the findings of study done in London by Nathan, et al in which $90 \%$ reference range was used in the analysis [12] This suggests that the range of conventional vital signs in the immediate postpartum period are similar across wide range of different population of postpartum women whether either $90 \%$ or $95 \%$ reference range is used in the analysis.

Physiologic changes of pregnancy leads to considerable haemodynamic adjustment in pregnant women [16]. Following the delivery of the placenta, auto-transfusion results in increase in cardiac output up to $80 \%$ above pre-pregnancy values [16]. It is in this haemodynamic adjustment in the immediate postpartum period that haemorrhage is most prevalent. Compensatory mechanisms can mask hypovolaemia and leads to little or no changes in conventional vital signs [17] This often leads to late detection of haemodynamic decompensation in postpartum women by healthcare providers leading to maternal morbidity and mortality as highlighted in the UK Confidential Enquiries into Maternal Deaths [3]. 
Shock index, first proposed as an early marker of haemodynamic instability in non-obstetric shock, has been studied extensively in non-specific shock, trauma, and sepsis as an earlier identifier of circulatory shock than conventional vital signs [18]. In a non-obstetric population the normal range has been defined and validated as 0.5 to 0.7 , with a $\mathrm{SI}>0.9$ indicating increased risk of mortality and morbidity $[12,13]$. In pregnancy, studies have suggested different normal ranges with 0.7-0.9 commonly cited in literature $[8,12]$ This range of shock index was derived from a study done by Le Bas, et al in which the mean SI was 0.74 with a range of $0.4-1.1$ [13]. Nathan, et al reported a range of $0.52-0.89$ in a secondary analysis of a prospective observational study of normal range of shock index in a London hospital [12]. In this study cohorts, the median shock index of 0.69 and $95 \%$ reference range of 0.48 - 0.89 supported the previous studies in which 0.9 is the upper limit of normal for postpartum women. This upper limit of 0.9 is higher than the upper limit of normal of 0.7 in non-obstetric population. The difference is due to the haemodynamic changes of pregnancy and delivery, mainly an increase in resting HR, which is often further increased during the immediate postpartum period owing to the stress of delivery. However, the lower limit of shock index of 0.48 in this study and that of previous study by Nathan, et al (0.52) [12] correspond to the lower limit of 0.5 for non-obstetric population. Although haemodynamic changes of pregnancy widens the normal shock index range, significant number $(n=101)$ of the participants maintained normal shock index within the reference range for non-obstetric population. The implication of shock index value lower than the lower limit currently used for obstetric population is still uncertain in clinical practice.

Socio-demographic and clinical interventions impacting on the haemodynamic physiology of pregnancy influence vital signs. In our maternity unit, active management of the third stage is routine and uterotonics are routinely administered as the newborn is delivered to prevent postpartum haemorrhage. In this study, oxytocin alone was used in the management of third stage so as to ensure uniformity. Ergometrine was avoided because it is usually contraindicated in those with hypertension, owing to its hypertensive effects. Therefore, effect of oxytocin on the vital signs was not tested in the linear logistic regression analysis even though it causes hypotension and tachycardia. In women receiving either epidural or spinal analgesia or anaesthesia, blood pressure can be reduced due to sympathetic block [19] Use of epidural analgesia is not routine in our maternity unit and analgesia in labour is achieved with either parenteral pentazocine or tramadol and none of the study participants received epidural analgesia. All the study cohorts that had caesarean section were given spinal anaesthesia. The effect of spinal anaesthesia on shock index was not factored in the regression analysis. Nathan, et al in their study showed that spinal anaesthetic use was not associated with a change in SI [12]. Although statistically significant, hypertensive disorders of pregnancy still maintain SI within normal range in this study. Also, maternal anaemia at the time of delivery did not have effect on the SI in this study. Previous study have shown that maternal hypertension and anaemia have little influence on SI in postpartum women [12]. Unlike blood pressure, pulse rate and mean arterial pressure that were significantly affected by maternal demographic and obstetric characteristics, these factors have no effect on shock index in this study cohorts. Therefore, SI does not need to be altered in the presence or absence of these factors. 
The strength of this study is that it is the first prospective study to determine the reference ranges of shock index and other conventional vital signs at one hour postpartum in women with normal blood loss at delivery in low resource setting to aid timely intervention in deteriorating postpartum women. However, the limitation of the study is that the participants were of Igbo ethnicity and the mono-ethnic nature of the cohorts may limit the generalisability of the findings of this study. In addition, one point assessment was done in this study. The authors recommend that serial measurement of shock index at intervals postpartum should be done in future studies to determine whether shock index values varies significant in women with normal blood loss at delivery.

In conclusion, the upper limit of obstetric shock index of 0.89 in well postpartum women in this study cohorts did support the current literature suggesting a threshold of 0.9 as upper limit of normal. However, the lower limit of shock index of 0.48 in this study corresponds to the lower limit of 0.5 for non-obstetric population. The authors recommend a change of obstetric shock index normal range from $0.7-0.9$ to 0.5 -0.9 to accommodate this lower threshold. This expanded range of obstetric shock index (0.5 - 0.9$)$ should be used to monitor postpartum women in all settings.

\section{Declarations}

\section{Statement of Consent}

The purpose and process of the study were explained to all participants. They were informed that their participation was voluntary and that they could withdraw at any time for any reason without any penalty either personal or affecting their medical care. The study participants were required to sign informed consent form before they were allowed to participate in the study.

\section{Acknowledgement}

Nil

\section{Conflict of interest}

The authors declare no conflict of interest

\section{References}

1. World Health Organization. Maternal mortality. Available at https://www.who.int/news-room/factsheets/detail/maternal-mortality. Accessed July 5, 2019.

2. World Health Organization. Maternal health in Nigeria: generating information for action: Available at https://www.who.int/ reproductivehealth/ maternal-health- nigeria/en/. Accessed July 5, 2019.

3. Knight M, Kenyon S, Brocklehurst P, Neilson J, Shakespeare J, Kurinczuk J. Saving lives, improving mothers' care - lessons learned to inform future maternity care from the UK and Ireland Confidential 
Enquiries into Maternal Deaths and Morbidity 2009 - 12.: MBRRACE-UK, Oxford: National Perinatal Epidemiology Unit, University of Oxford 2014.

4. Say L, Chou D, Gemmill A, Tuncalp O , Moller A-B, Daniels J, et al. Global causes of maternal death: a WHO systematic analysis. Lancet Glob Health. 2014; 2(6): e323 - e33. doi: 10.1016/S2214-109X(14) 70227-X PMID: 25103301.

5. Schorn MN. Measurement of blood loss: review of the literature. Journal of Midwifery Women's Health. 2010; 55(1): $20-7$.

6. Schoon M, editor Proposed colour banded early warning observation charts for South Africa: review. Obstetrics and Gynaecology Forum; 2012: Sabinet Online.

7. Birkhahn RH, Gaeta TJ, Van Deusen SK, Tloczkowski J. The ability of traditional vital signs and shock index to identify ruptured ectopic pregnancy. Am J Obstet Gynecol. 2003; 189(5): 1293 - 6. PMID: 14634556.

8. Nwafor JI, Obi VO, Onwe BI, Ugoji DPC, Onuchukwu VU, Obi CN, et al. Comparison of performance of shock index and conventional vital sign parameters for prediction of adverse maternal outcomes following major postpartum hemorrhage in Abakaliki, Southeast, Nigeria. Trop J Obstet Gynaecol 2019; 36(3): 431 - 436.

9. Paladino L, Subramanian RA, Nabors S, Sinert R. The utility of shock index in differentiating major from minor injury. Eur J Emerg Med. 2011; 18(2): 94-8. doi: 10.1097/MEJ.0b013e32833f212b PMID: 20842040.

10. Rady MY, Nightingale P, Little RA, Edwards JD. Shock index: a re-evaluation in acute circulatory failure. Resuscitation. 1992; 23(3): 227 - 34. PMID: 1321482

11. Asaari $\mathrm{H}$. Value of shock index in prognosticating the short term outcome of death for patients presenting with severe sepsis and septic shock in the emergency department. Med J Malaysia. 2012; 67(4): 407.

12. Nathan HL, Cottam K, Hezelgrave NL, Seed PT, Briley A, Bewley S, et al. Determination of normal ranges of shock index and other haemodynamic variables in the immediate postpartum period: $A$ cohort study. PLoS ONE, 2016 11(12), [e0168535]. https://doi.org/10.1371/journal.pone.0168535.

13. Le Bas A, Chandraharan E, Addei A, Arulkumaran S. Use of the obstetric shock index as an adjunct in identifying significant blood loss in patients with massive postpartum hemorrhage. Int J Gynecol Obstet. 2014 (4); 124: 253 - 5.

14. Taylor D, Fleischer A, Meirowitz N, Rosen L. Shock index and vital-sign reference ranges during the immediate postpartum period. Int J Gynecol Obstet. 2017; 137(2): 192-5.

15. World Health Organisation. WHO recommendations on Postnatal care of the mother and newborn. 2013.

16. Robson S, Dunlop W, Hunter S. Haemodynamic changes during the early puerperium. BMJ. 1987; 294(6579): 1065. PMID: 3107696.

17. Robson SC, Boys RJ, Hunter S, Dunlop W. Maternal hemodynamics after normal delivery and delivery complicated by postpartum hemorrhage. Obstet Gynecol. 1989; 74(2): 234 - 9. PMID: 2787489. 
18. Nathan HL, El Ayadi A, Hezelgrave NL, Seed P, Butrick E, Miller S, et al. Shock index: an effective predictor of outcome in postpartum haemorrhage? BJOG. 2105; 122(2): 268-75.

19. Holte K, Foss NB, SvenseÂn C, Lund C, Madsen JL, Kehlet H. Epidural anesthesia, hypotension, and changes in intravascular volume. Anesthesiology. 2004; 100(2): 281 - 6. PMID: 14739801

\section{Tables}

Table 1: Study cohorts characteristics $(\mathrm{N}=181)$

\begin{tabular}{|c|c|}
\hline Characteristics & Frequency (\%) \\
\hline \multicolumn{2}{|l|}{ Age (years) } \\
\hline $15-24$ & $20(11.05)$ \\
\hline $25-34$ & $123(67.96)$ \\
\hline $35-44$ & $38(20.99)$ \\
\hline Mean age (years) $\pm S D$ & $30.19 \pm 5.2$ \\
\hline \multicolumn{2}{|l|}{ Parity } \\
\hline $0-1$ & $99(54.7)$ \\
\hline $2-3$ & $55(30.39)$ \\
\hline $4-5$ & $17(9.39)$ \\
\hline $6-7$ & 7 (3.87) \\
\hline $8-9$ & $3(1.66)$ \\
\hline Mean parity \pm SD & $1.79 \pm 1.9$ \\
\hline Mean BMI $\left(\mathrm{kg} / \mathrm{m}^{2}\right) \pm \mathrm{SD}$ & $27.48 \pm 5.5$ \\
\hline Singleton pregnancy & $176(97.2)$ \\
\hline Multiple pregnancy & $5(2.8)$ \\
\hline Vaginal (including instrumental) delivery & $94(51.93)$ \\
\hline Caesarean section & 87 (48.07) \\
\hline Hypertensive disorders of pregnancy & $36(19.89)$ \\
\hline Anaemia $(\mathrm{Hb}<10 \mathrm{~g} / \mathrm{dl})$ at delivery & $3(1.66)$ \\
\hline Median [IQR] blood loss, ml & $300[200-400]$ \\
\hline
\end{tabular}

Table 2: Median, lower and upper quartile and 95\% reference ranges for systolic blood pressure, diastolic blood pressure, mean arterial pressure, heart rate and shock index

\begin{tabular}{lccc} 
Tariable & Median (IQR) & \multicolumn{2}{c}{$95 \%$ Reference range } \\
\cline { 3 - 4 } & & Lower limit (90\%CI) & Upper limit (90\%CI) \\
\hline Jeart rate (bpm) & $82(80-90)$ & $65(63-67)$ & $102(100.6-104.6)$ \\
iystolic BP (mmHg) & $120(110-130)$ & $100(99.8-102)$ & $155.8(152.3-159.4)$ \\
)iastolicBP (mmHg) & $70(70-80)$ & $60(59.8-60.6)$ & $94(92.1-96.6)$ \\
Mean arterial pressure (mmHg) & $90(83.3-96.7)$ & $66.5(63.8-69.2)$ & $116.6(113.9-119.3)$ \\
ihock index & $0.69(0.63-0.75)$ & $0.48(0.46-0.5)$ & $0.89(0.87-0.9)$ \\
\hline
\end{tabular}


IQR = Interquartile range, $\mathrm{CI}=$ Confidence interval

Table 3: Linear regression analysis showing association between shock index and demographic and obstetric characteristics

\begin{tabular}{|c|c|c|c|c|c|}
\hline \multirow{2}{*}{$\begin{array}{l}\text { Variables } \\
\text { Age(years) }\end{array}$} & Coefficient & \multicolumn{2}{|c|}{ Standard error } & \multirow{2}{*}{$\frac{95 \% \text { CI }}{-0.005-0.001}$} & \multirow{2}{*}{$\frac{\text { P-value }}{0.112}$} \\
\hline & -0.002 & 0.001 & -1.599 & & \\
\hline Parity & 0.005 & 0.004 & 1.174 & $-0.003-0.013$ & 0.242 \\
\hline Weight(Kg) & -0.003 & 0.003 & -0.972 & $-0.008-0.003$ & 0.332 \\
\hline Height(meters) & 0.269 & 0.287 & 0.938 & $-0.297-0.836$ & 0.349 \\
\hline $\operatorname{BMI}\left(\mathrm{kg} / \mathrm{m}^{2}\right)$ & 0.006 & 0.007 & 0.886 & $-0.008-0.020$ & 0.377 \\
\hline Anaemia $(<10 \mathrm{~g} / \mathrm{dl})$ & -0.011 & 0.061 & -0.180 & $-0.132-0.110$ & 0.858 \\
\hline Multiple gestation & -0.078 & 0.076 & -1.028 & $-0.228-0.072$ & 0.305 \\
\hline Singleton pregnancy & 0.002 & 0.078 & 0.028 & $-0.152-0.157$ & 0.978 \\
\hline Hypertensive disorders & -0.106 & 0.018 & -5.742 & $-0.142-(-0.069)$ & $<0.001$ \\
\hline Mode of delivery & -0.027 & 0.015 & -1.877 & $-0.056-0.001$ & 0.062 \\
\hline
\end{tabular}

Table 4: Linear regression analysis showing association between systolic blood pressure and demographic and obstetric characteristics

\begin{tabular}{lccccc}
\hline Variables & Coefficient & Standard error & $\mathrm{t}$ & $95 \%$ CI & P-value \\
\hline Age(years) & 0.231 & 0.231 & 1.481 & $-0.077-0.540$ & 0.140 \\
Parity & -0.286 & 0.446 & -0.642 & $-1.166-0.593$ & 0.522 \\
Weight(Kg) & -0.142 & 0.314 & -0.451 & $-0.761-0.478$ & 0.652 \\
Height(meters) & -0.225 & 31.645 & -0.007 & $-62.69-62.24$ & 0.994 \\
BMI(kg/m ${ }^{2}$ ) & 0.431 & 0.789 & 0.547 & $-1.125-1.988$ & 0.585 \\
Anaemia(<10g/dl) & 3.577 & 6.753 & 0.530 & $-9.753-16.907$ & 0.597 \\
Multiple gestation & 19.225 & 8.375 & 2.295 & $2.692-35.757$ & 0.023 \\
Singleton pregnancy & 7.803 & 8.630 & 0.904 & $-9.233-24.838$ & 0.367 \\
Hypertensive disorders & 31.640 & 2.031 & 15.576 & $27.630-35.650$ & $<0.001$ \\
Mode of delivery & 2.419 & 1.605 & 1.507 & $-0.750-5.588$ & 0.134 \\
\hline
\end{tabular}

Table 5: Linear regression analysis showing association between diastolic blood pressure and demographic and obstetric characteristics 


\begin{tabular}{lccccc}
\hline Variables & Coefficient & Standard error & $\mathrm{t}$ & $95 \%$ CI & P-value \\
\hline Age(years) & 0.285 & 0.129 & 2.204 & $0.03-0.54$ & 0.029 \\
Parity & -0.629 & 0.368 & -1.707 & $-1.356-0.098$ & 0.090 \\
Weight(Kg) & -0.096 & 0.259 & -0.371 & $-0.608-0.416$ & 0.711 \\
Height(meters) & -7.755 & 26.162 & -0.296 & $-59.39-43.889$ & 0.767 \\
BMI(kg/m $\left.{ }^{2}\right)$ & 0.102 & 0.652 & 0.156 & $-1.186-1.389$ & 0.876 \\
Anaemia(<10g/dl) & 9.741 & 5.583 & 1.745 & $-1.279-20.761$ & 0.083 \\
Multiple gestation & 19.643 & 6.924 & 2.837 & $5.975-33.311$ & 0.005 \\
Singleton pregnancy & 11.763 & 7.135 & 1.649 & $-2.321-25.846$ & 0.101 \\
Hypertensive disorders & 19.921 & 1.679 & 11.863 & $16.60-23.23$ & $<0.001$ \\
Mode of delivery & -1.520 & 1.327 & -1.145 & $-4.140-1.100$ & 0.254 \\
\hline
\end{tabular}

Table 6: Linear regression analysis showing association between heart rate and demographic and obstetric characteristics

\begin{tabular}{lccccc}
\hline Variables & Coefficient & Standard error & $\mathrm{t}$ & $95 \%$ CI & P-value \\
\hline Age(years) & -0.124 & 0.141 & -0.875 & $-0.403-0.155$ & 0.383 \\
Parity & 0.441 & 0.403 & 1.095 & $-0.354-1.237$ & 0.275 \\
Weight(Kg) & -0.359 & 0.284 & -1.266 & $-0.920-0.201$ & 0.207 \\
Height(meters) & 28.624 & 28.625 & 1.000 & $-27.88-85.12$ & 0.319 \\
BMI(kg/m ${ }^{2}$ ) & 0.975 & 0.713 & 1.367 & $-0.433-2.384$ & 0.173 \\
Anaemia(<10g/dl) & 0.100 & 6.108 & 0.016 & $-11.95-12.15$ & 0.987 \\
Multiple gestation & 0.400 & 7.576 & 0.053 & $-14.55-15.35$ & 0.958 \\
Singleton pregnancy & 3.746 & 7.806 & 0.480 & $-11.66-19.15$ & 0.632 \\
Hypertensive disorders & 6.408 & 1.837 & 3.488 & $2.781-10.035$ & 0.001 \\
Mode of delivery & -1.359 & 1.452 & -0.936 & $-4.22-1.507$ & 0.351 \\
\hline
\end{tabular}

Table 7: Linear regression analysis showing association between mean arterial pressure and demographic and obstetric characteristics 


\begin{tabular}{lccccc}
\hline Variables & Coefficient & Standard error & $\mathrm{t}$ & $95 \%$ CI & P-value \\
\hline Age(years) & 0.272 & 0.122 & 2.238 & $0.032-0.513$ & 0.027 \\
Parity & -0.538 & 0.347 & -1.551 & $-1.224-0.147$ & 0.123 \\
Weight(Kg) & -0.108 & 0.245 & -0.443 & $-0.591-0.374$ & 0.659 \\
Height(meters) & -5.424 & 24.654 & -0.220 & $-54.09-43.24$ & 0.826 \\
BMI(kg/m ${ }^{2}$ ) & 0.207 & 0.614 & 0.337 & $-1.006-1.420$ & 0.736 \\
Anaemia(<10g/dl) & 7.705 & 5.261 & 1.465 & $-2.68-18.09$ & 0.145 \\
Multiple gestation & 19.442 & 6.525 & 2.980 & $6.561-32.322$ & 0.003 \\
Singleton pregnancy & 10.496 & 6.724 & 1.561 & $-2.776-23.768$ & 0.120 \\
Hypertensive disorders & 23.774 & 1.583 & 15.023 & $20.65-26.898$ & $<0.001$ \\
Mode of delivery & -0.138 & 1.251 & -0.110 & $-2.607-2.331$ & 0.912 \\
\hline
\end{tabular}

Figures

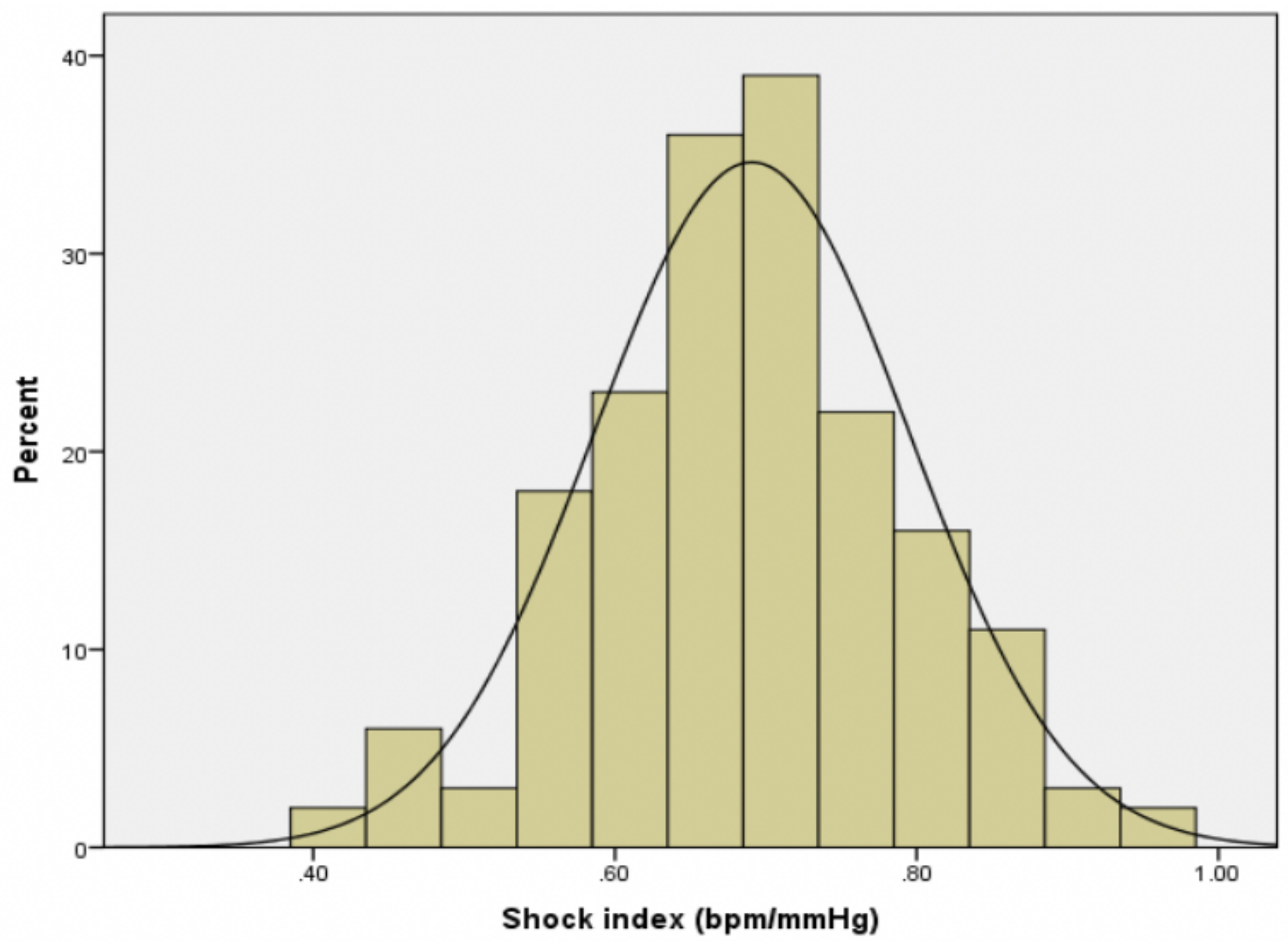

Figure 1 
Histogram of distribution of shock index values with superimposed normal distribution curve.

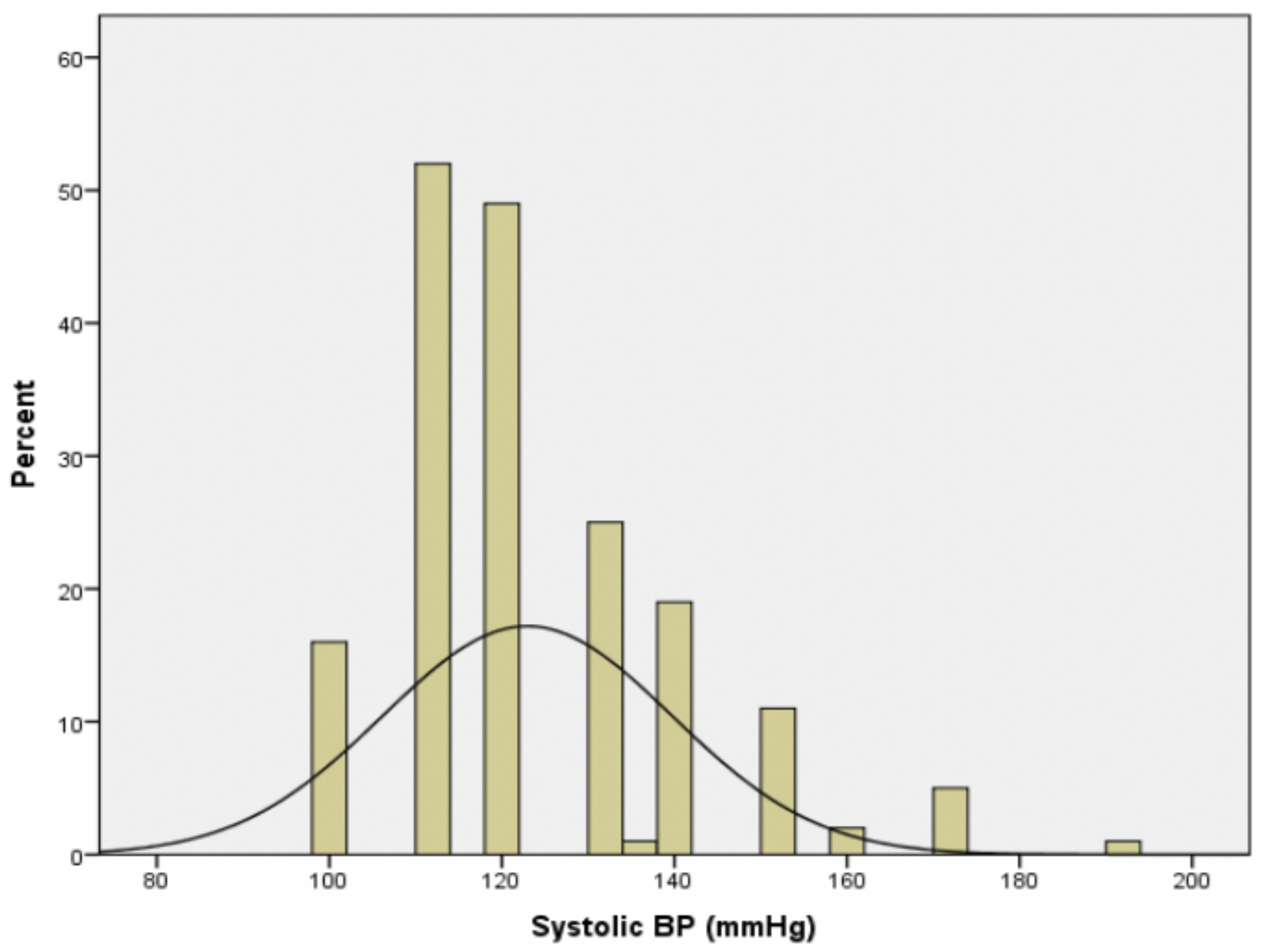

Figure 2

Histogram of distribution of systolic blood pressure values with superimposed normal distribution curve. 


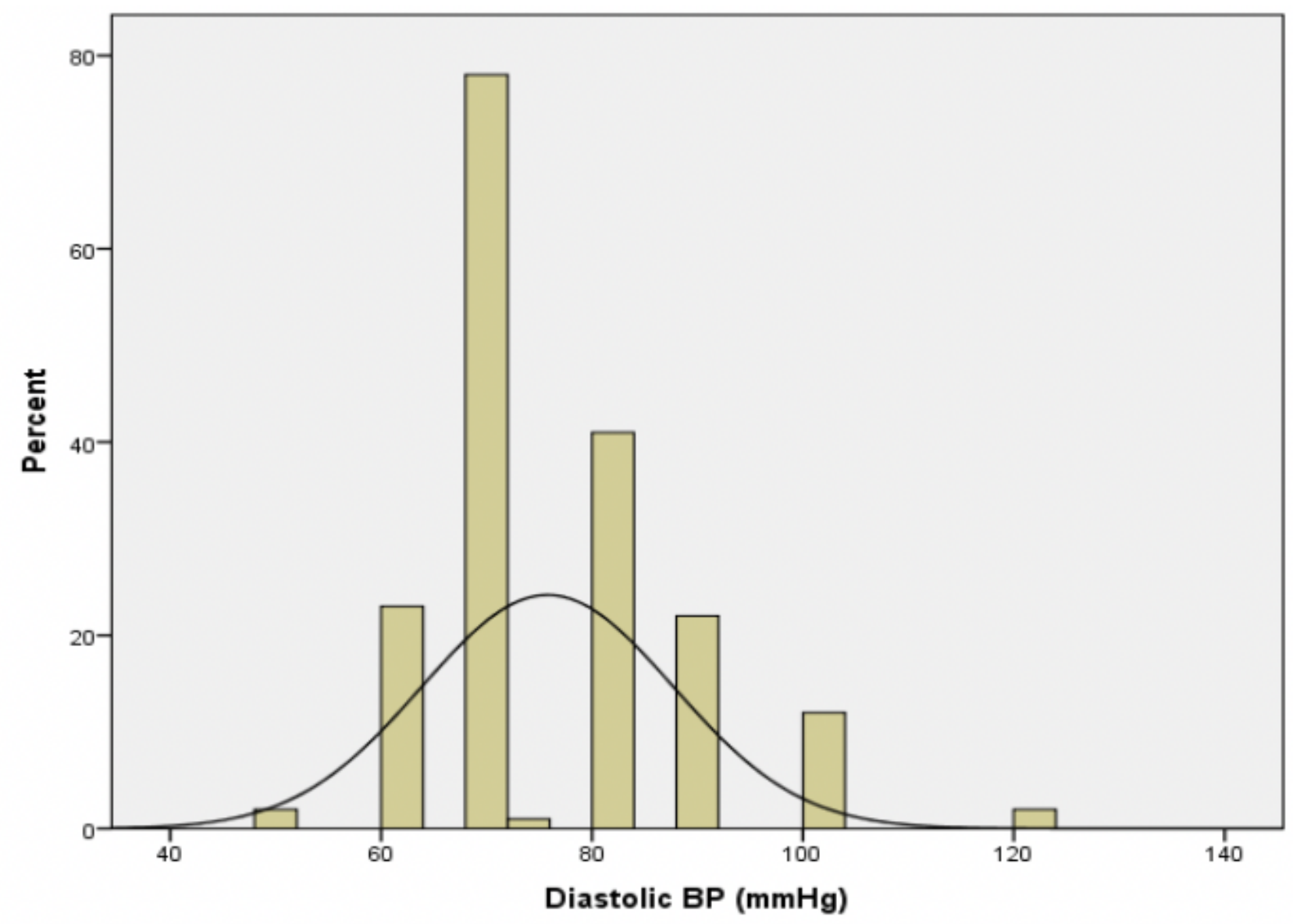

Figure 3

Histogram of distribution of diastolic blood pressure values with superimposed normal distribution curve. 


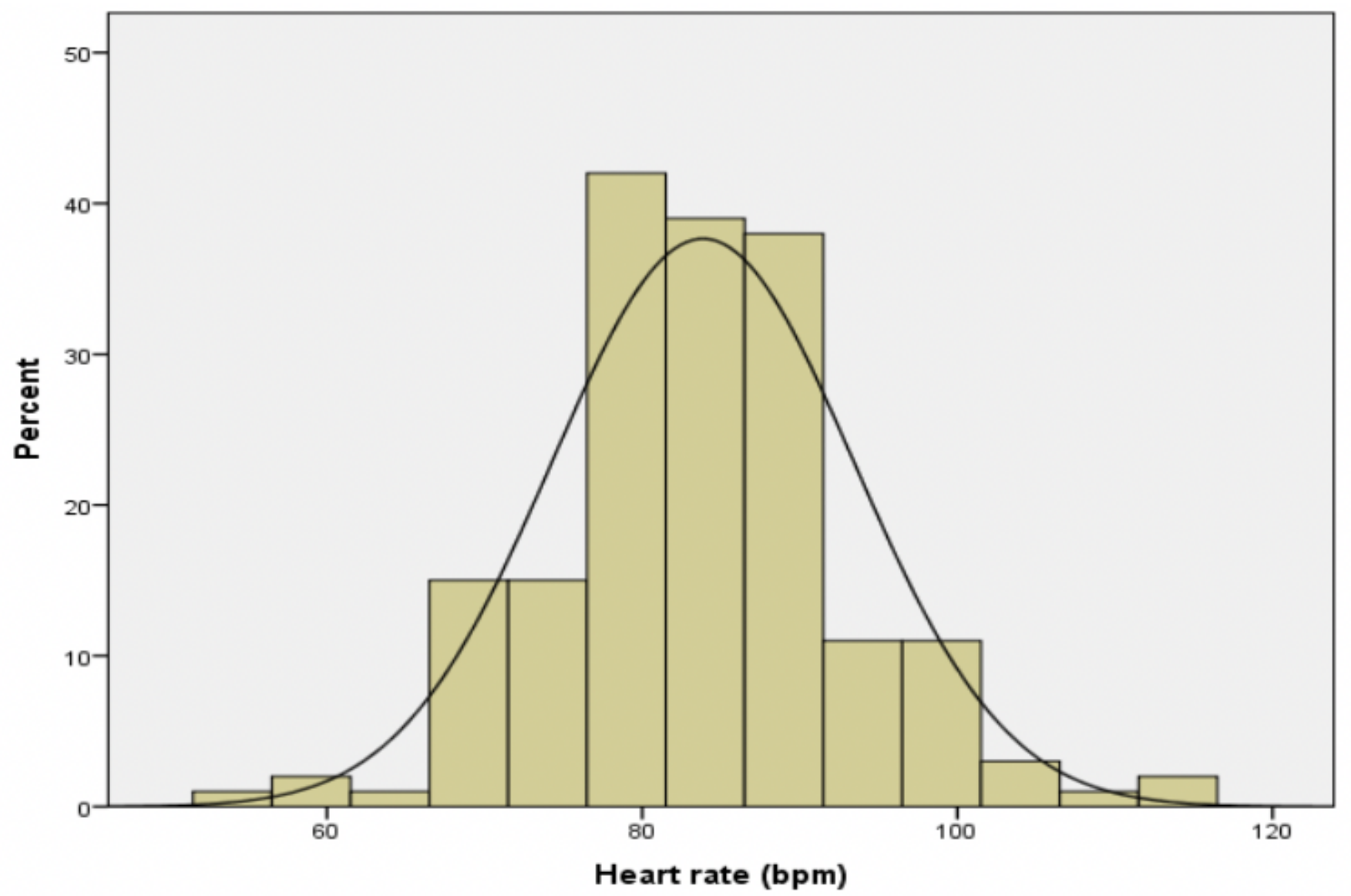

Figure 4

Histogram of distribution of heart rate values with superimposed normal distribution curve. 


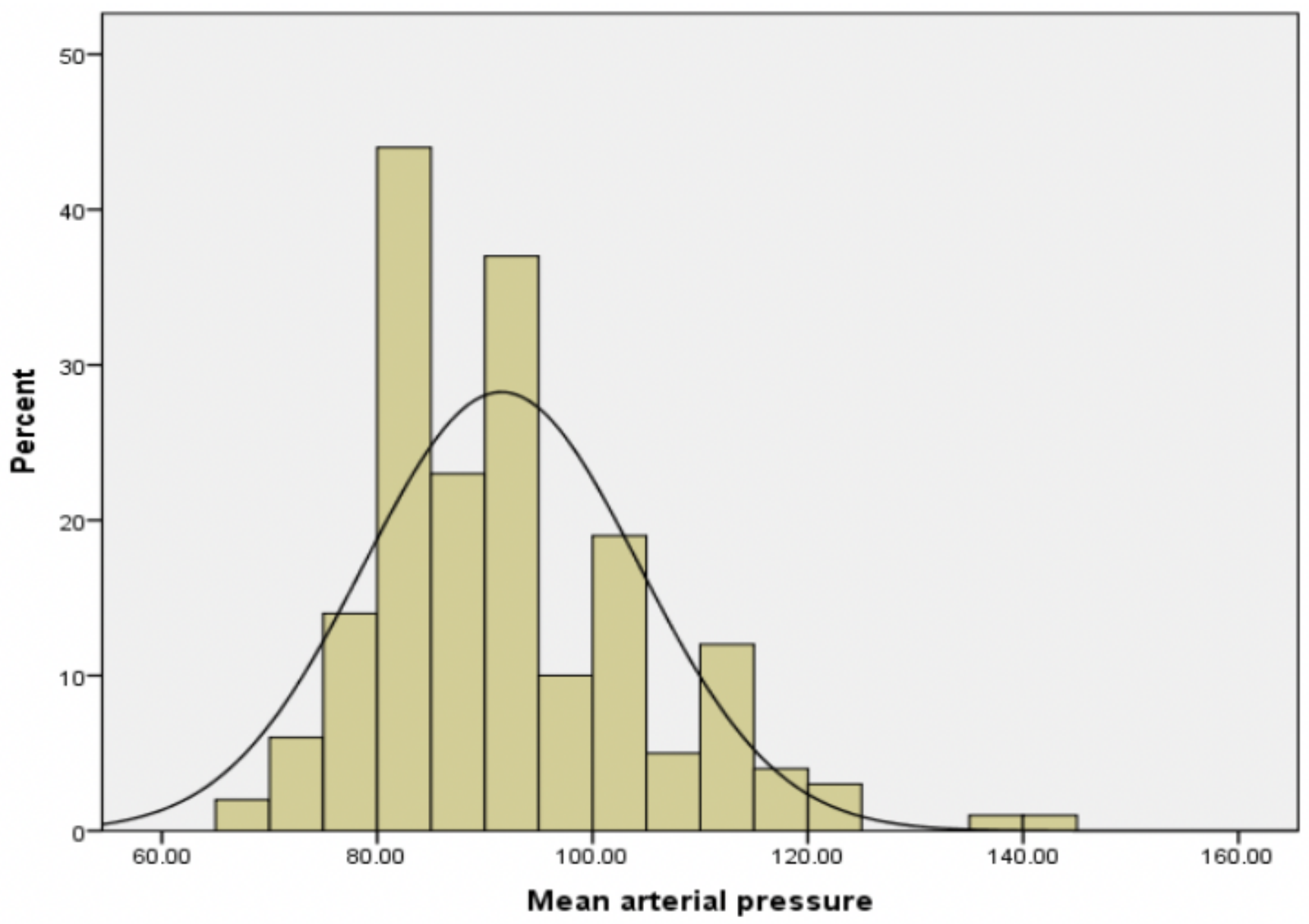

Figure 5

Histogram of distribution of mean arterial pressure values with superimposed normal distribution curve. 